\title{
The Effective Sequence of Uniformities and its Limit as a Methodology in Computable Analysis*
}

\author{
Mariko YASUGI, Takakazu MoRI and Yoshiki TsUJII ${ }^{\dagger}$
}

\begin{abstract}
We will speculate on the theory of the effective sequence of unifomities on a set and its effective limit as a methodology to vest a sequence of real functions which have different points of discontinuities with some notion of computability. Some model examples which explain the necessity of such a methodology are presented.
\end{abstract}

\section{Introduction and background}

The standard notion of computability of a real number or of a sequence of real numbers as well as that of computability of a real continuous function or of a sequence of continuous functions is now generally agreed (cf. Section 2). There are many references on this subject. We refer the reader to [10]; there is also [23] for a quick read.

There are many familiar functions which are discontinuous at some points and to which we would like to attribute a certain kind of computability. One approach to this problem was proposed by Pour-El and Richards [10] and was succeeded by Washihara (cf. [14], [15], [16]) and others. It was a functional analysis approach. In their treatment, a function is regarded as computable as a point in some function space. This is sufficient when one draws a rough graph of the function, but does not supply us with information how to compute individual values.

In an attempt to compute a discontinuous function, a problem arises in the computation of the value at a jump point (a point of discontinuity). Suppose a number $a$ is a jump point. The question " $x=a$ ?" is in general not decidable even for computable $x$ and $a$. This implies that one cannot decide whether a given number $x$ is a jump point, which in turn implies that there is no computation algorithm to compute the value of a function at a jump point. Very often, however, we do compute the value of a function at a point of discontinuity.

There are many ways of characterizing computation of a discontinuous function. We have proposed two approaches to this problem. One is to represent the value of

* This work has been supported in part by Kayamori Foundations of Informational Sciences Advancent K15VIII No. 157 and Science Foundations of JSPS No. 16340028.

+ E-mail : yasugi, morita, tsujiiy@ cc.kyoto-su.ac.jp 
a function at a jump point in terms of a recursive rational sequence approximating it with a "limiting recursive modulus of convergence" instead of a recursive one (Yasugi, Brattka, Washihara: [18]). (A limiting recursive function is obtained from a recursive function by taking the limit of its values if the limit exists. The notion of the limiting recursive function is due to Gold [2], and has been utilized also in some works related to ours: cf. [9] and [3].) Another is to change the topology of the domain of a function (Tsujii, Mori, Yasugi : [12]) so that a discontinuous function (in the Euclidean topology) becomes continuous in the new topology. More precisely, we obtain a "uniform topology" by "isolating" the jump points. Under certain conditions, these two approaches are equivalent ([21]).

Let us explain the problem and the idea of isolation in more detail with the case of computing $[x]$, the Gaußian function. This example has been extensively taken up in [18], and helps to distill the situation.

The Gaußian function $[x]$ outputs the "greatest integer" that does not exceed $x$. It can be defined as

$$
[x]=n \quad \text { if } \quad n \leq x<n+1
$$

for each integer $n$, and hence the value can be determined by judging $<$ alone unless $x$ be an integer. When $x$ is an integer, $x=n$ ? is usually undecidable (even for a computable $x$ ), and hence there is no general computation algorithm for $[x]$. (A counter-example is given in [18].)

From what sort of viewpoint can one discuss the computability of a function like this?

Let $x$ be a computable real number, and let us attempt to compute its value $[x]$. For the sake of simplicity, we assume $x>0$.

For $n=0,1,2,3, \cdots$, keep asking " $x<n$ ?" and " $n<x$ ?" (Technically, we try to decide the inequality with respect to a recursive sequence of rational numbers, say $\left\{r_{m}\right\}$, which approximates $x$ with a recursive modulus of convergence, say $\alpha$ : cf. Section 2.) One will infallibly hit an $n$ satisfying $n<x<n+2$. If one is fortunate so that one hits an $n$ satisfying $n<x<n+1$, then one can put $[x]=n$ and the computation halts. In general, however, one cannot decide whether $n<x<n+1$ or $n+1 \leq x<n+2$.

So, one checks the inequality

$$
r_{\alpha(p)}<(n+1)-\frac{1}{2^{p}}
$$

for $p=1,2, \cdots$. According to its answer, we define a sequence of integers $\left\{N_{p}\right\}$ as follows.

While the answer is $N o$, put $N_{p}=n+1$. Once the answer becomes Yes at $p$, then put $N_{q}=n$ for all $q$ satisfying $q \geq p . \quad\left\{N_{p}\right\}$ is a well-defined and recursive 
sequence of rationals (integers, as a matter of fact), and it can be shown that, if $N_{p}=$ $n+1$ holds for all $p$, then the limit of the sequence is $n+1$; otherwise, the limit is $n$. In either case, the sequence $\left\{N_{p}\right\}$ approximates the value $[x]$.

We have to be careful here to note that it is not decidable whether the limit is $n$ or $n+1$. It is true that one of the two cases definitely holds, and the limit is the value $[x]$. Furthermore, for each case, there is a recursive modulus of convergence. Only we do not know which case holds.

This undecidability indicates that a master program to compute $[x]$ is not guaranteed. Indeed, there is a computable sequence of real numbers $\left\{x_{n}\right\}$, where the sequence of its values $\left\{\left[x_{n}\right]\right\}$ does not form a computable sequence of reals (integers, in this case : cf. [18]).

On the other hand, in particular, we do distinguish an integer $n$ on the real line and easily compute $[n](=n)$. Then, excluding integers, we can run a program to compute $[x]$.

How can we express such an intellectual activity of the human being? This was the origin of our study of "effective uniformity" in relation to the computability problem in analysis. One interpretation was proposed in [18]. Namely, one allows the outputs $\left\{\left[x_{n}\right]\right\}$ to be approximated by a recursive double sequence of rationals with a "limiting recursive" modulus of convergence, and its meaning was speculated in [17]. This is mathatically interesting and significant, but does not really represent our mental activity. We have found that most natural mathematical representation of such an activity is "change of topology" of the real line, the domain of a function, by isolating the jump points. In the case of the Gaußian function $[x]$, one proceeds as follows.

Isolate each integer $n$ so that the fundamental neighborhood system of the point $n$ consists of the singleton $\{n\}$ alone, while each open interval $(n-1, n)$ preserves the Euclidean topology. The collection $\{\{n\},(n-1, n): n=1,2,3, \cdots\}$ induces a "uniform topology." As far as the computability of a function is concerned, there is no need to evaluate the metric of the domain, and hence assigning the uniform topology suffices.

With respect to the thus obtained uniform topology, the function $[x]$ becomes continuous, since there is no element in the neighborhood of an integer $x=n$ other than $n$. By assigning "effectivity" to this uniform space, we can develop a theory of computability of a function such as $[x]$ as the computability theory of the continuous functions (cf. [12]). In this treatment, the uniform space as above has been associated with the function $[x]$. In fact, this topology is the "weakest topology" for the function $[x]$ to become continuous. We would call it the "natural topology" for $[x]$. (Technically, a topology $\mathcal{T}$ on a set $X$ is called the weakest for a function $f: X \rightarrow Y$ if the inverse image of the open sets of $Y$ by $f$ forms the system of fundamental neighborhoods for $\mathcal{T}$.) 
In analysis, the limit of a function sequence $\left\{f_{n}\right\}$ plays an important role. In such a case, to each function $f_{n}$ a uniform topology must be assigned, since the functions in the sequence may have different jump points. On the other hand, in order to study the limit function of the sequence, all the functions must be treated in one topology. We have thus been led to the notion of the "effective limit" of a sequence of uniform spaces.

As for the "sequence" of functions with different jump points, we first proposed an approach, guided by an example of the system of Rademacher functions, in terms of the "limiting recursive modulus of convergence" ([24]). Let $\left\{\phi_{l}(x)\right\}$ be the sequence of Rademacher functions (cf. [11], [24]). In [24], it was shown that $\left\{\phi_{l}\right.$ $(x)\}$ has a "weak sequential computability," that is, there is a program which does the following job: input a computable sequence of real numbers $\left\{x_{m}\right\}$, a recursive triple sequence of rational numbers can be constructed so that it converges to $\left\{\phi_{l}\right.$ $\left.\left(x_{m}\right)\right\}$ with a limiting recursive modulus of convergence. This method, however, does not represent our mental activity of computing the values of a function sequence at the jump points, as was the case of a single function $[x]$. We have thus resorted to an alternative way, using the notion of the "effective sequence of uniformities."

In this article, we develop the theory of "effective sequence of uniformities" and its "effective limit" (Section 4) in order to realize the idea stated above. Under a certain condition, the "limit" of such an effective sequence is again an effective uniformity (Section 4: Theorem 1). Several examples supporting the theory of effective sequence of uniformities will be presented in Section 3. Effectivity of models of Section 3 is demonstrated in Section 6.

Our original theory of the effective uniform space can be seen in [12], [22] and [21].

We will first give a brief account of some known fundamental notions such as computable real numbers and computable (continuous) real functions as well as the effective uniformity and the computability structure on the effective uniform space (Section 2) for the reader's convenience.

In Section 5, we present a special case of the theory proposed in Section 4. There we confine ourselves to the real numbers in the interval $I=[0,1)$ and real functions on it. The theory of an effective sequence of uniformities on $I$ and its limit is developed (Propositions 5.1, 5.3). For the real sequences from $I, \mathbf{E}-$ computability (computability in the Euclidean topology), $\nu$-computability (computability with respect to the $\nu$-th uniformity in the sequence, $\nu=1,2,3, \cdots$ ) and $\omega$-computability (computability with respect to the limit of the sequence) will be defined, and their mutual relationships will be worked out (Propositions 5.2 5.3). This limit space is topologically equivalent to the Fine-metric space (cf. Remark in Section 8). 
In Section 7, a gerenal treatment of the computable sequence of functions on the sequence of uniformities will be presented. The functions in the models in Section 3 will then be discussed in Section 8 .

We only list some references which have close relationship with the present work.

We hope to modify our approach to a function sequence with points of discontinuity defined on a metric space with a computability structure (cf. [7] and [19]).

The article is composed in a manner that some model examples are first given, prior to the theory, so that the motivation for the theory can be better understood. It is made self-contained as much as possible.

\section{Preliminaries}

In the following, $\mathbf{R}$ will denote the set of real numbers. In general, letters such as $p, l, m, n, k, \mu, \nu$ will be used to denote positive integers or natural numbers.

For the subsequent discussion, let us here note the following fundamental facts: $=, \leq,<$ on natural numbers and on rational numbers are decidable; $a<b$ can be decided for computable real numbers $a$ and $b$ when the inequality indeed holds, while $a=b$ and $a \leq b$ are not necessarily decidable even for computable real numbers $a$ and $b$.

We will first introduce some basic definitions.

Definition 2.1 (Euclidean computability: cf. [10], [23]) 1) A sequence of rational numbers $\left\{r_{n}\right\}$ is called recursive if

$$
r_{m}=(-1)^{\beta(m)} \frac{\gamma(m)}{\delta(m)}
$$

hold with recursive functions $\beta, \gamma$ and $\delta$.

2) A sequence of real numbers $\left\{x_{i}\right\}$ is called $\mathbf{E}$-computable (computable with respect to the Euclidean topology) if

$$
\forall m \geq \alpha(i, p) .\left|x_{i}-r_{i m}\right|<\frac{1}{2^{p}}
$$

for a recursive function $\alpha$ and a recursive sequence of rational numbers $\left\{r_{i m}\right\}$. This property will be expressed as $x_{i} \simeq_{\mathbf{E}}\left\langle r_{i m}, \alpha(i, p)\right\rangle$, or, for short, $x_{i} \simeq_{\mathbf{E}}\left\langle r_{i m}, \alpha\right\rangle$.

A real number $x$ is called $\mathbf{E}$-computable if $\{x, x, x, \cdots\}$ is.

3) A real (continuous) function $f$ is computable (E-computable) if the following (i) and (ii) hold.

(i) $f$ preserves sequential computability, that is, for a computable $\left\{x_{n}\right\},\left\{f\left(x_{n}\right)\right\}$ is computable.

(ii) $f$ is effectively continuous with a recursive $\beta$ :

$$
-103-
$$




$$
\forall p \forall n \forall k \geq \beta(n, p) \forall x, y \in[-n, n] .|x-y|<\frac{1}{2^{k}} \Rightarrow|f(x)-f(y)|<\frac{1}{2^{p}} .
$$

This definition can be extended to a sequence of functions.

Remark Notice that, in the definition above, the Euclidean computability of a function is defined only for a continuous function. On the other hand, in computing values of a piecewise continuous real function, for example, it is a common practise to first compute the value at a jump point, and then compute values on the open interval where the function is continuous. Such an action corresponds to the mathematical notion of isolating the jump points or isolating each half-closed interval. For instance, consider the function $f$ such that $f(x)=0$ if $x<0$ and $f(x)=$ 1 if $x \geq 0$, and isolate the intervals $I_{1}=(-\infty, 0)$ and $I_{2}=[0, \infty)$ from each other. Then we can regard $f$ as continuous on each interval of $I_{1}$ and $I_{2}$. We were thus led to the uniform topology of the real line induced from the Euclidean topology by isolating the jump points or the intervals with the jump points at ends. (We have employed the definition of uniformity as defined in [4].) For details of three definitions below, see [12] and [21].

Let $X$ be a non-empty set. A sequence $\left\{V_{n}\right\}_{n \in \mathrm{N}}$ of maps from $X$ to the powerset of $X$, that is, $V_{n}: X \rightarrow P(X)$, is called a (countable) uniformity if it satisfies some axioms, that is, Axioms $A_{1} \sim A_{5}$ to be stated below. Let us note that, in fact, $A_{1}$ and $A_{2}$ in [4] can be unified to $A_{1} \& A_{2}: \cap_{n} V_{n}(x)=\{x\}$. We will state Axioms $A_{3} \sim A_{5}$ in the form of effective uniformity.

Definition 2.2 (Effective uniformity : [12]) Let $\left\{V_{n}\right\}$ be as above. It is called an effective uniformity if there are recursive functions $\alpha_{1}, \alpha_{2}$ and $\alpha_{3}$ such that

$$
\begin{gathered}
\left(A_{1} \& A_{2}\right) \quad \cap_{n} V_{n}(x)=\{x\} ; \\
\text { (Effective } \left.A_{3}\right) \quad \forall n, m \forall x \in X . V_{\alpha_{1}(n, m)}(x) \subseteq V_{n}(x) \cap V_{m}(\mathrm{x}) ; \\
\text { (Effective } \left.A_{4}\right) \quad \forall n \forall x, y \in X . x \in V_{\alpha_{2}(n)}(y) \Rightarrow y \in V_{n}(x) ; \\
\text { (Effective } A_{5} \text { ) } \forall n \forall x, y, z \in X . x \in V_{\alpha_{s}(n)}(y) \wedge y \in V_{\alpha_{s}(n)}(z) \Rightarrow x \in V_{n}(z) .
\end{gathered}
$$

The system $\mathcal{T}:=\left\langle X,\left\{V_{n}\right\}, \alpha_{1}, \alpha_{2}, \alpha_{3}\right\rangle$ will be called an effective uniform topological space.

Note It is known that $\left\{V_{n}(x)\right\}$ forms a system of fundamental neighborhoods of $x$ for $x \in X$.

Definition 2.3 (Effective $V$-convergence : [12]) A sequence $\left\{x_{k}\right\}$ from $X$ is said to effectively $V$-converge to $x$ in $X$ if there is a recursive function $\gamma$ satisfying

$$
\begin{gathered}
\forall n \forall k \geq \gamma(n) . x_{k} \in V_{n}(x) . \\
-104-
\end{gathered}
$$


This property will be expressed as $x \simeq_{v}\left\langle x_{k}, \gamma\right\rangle$.

This definition can be extended to effective convergence of a multiple sequence to a sequence.

Definition 2.4 (Computability structure: [12]) Let $\mathcal{S}$ be a family of sequences from $X$ (multiple sequences included). $\mathcal{S}$ is called a computability structure for $\mathcal{T}$ if the following $\mathrm{C} 1 \sim \mathrm{C} 3$ hold.

$\mathrm{Cl}$ : (Non-emptiness) $\mathcal{S}$ is nonempty.

C2: (Re-enumeration) If $\left\{x_{k}\right\} \in \mathcal{S}$ and $\alpha$ is a recursive function, then $\left\{x_{\alpha(i)}\right\}_{i} \in$ $\mathcal{S}$.

C3: (Limit) If $\left\{x_{l k}\right\}$ belongs to $\mathcal{S},\left\{x_{l}\right\}$ is a sequence from $X$, and $\left\{x_{l k}\right\} V$ converges to $\left\{x_{l}\right\}$ effectively as $k$ tends to the infinity, then $\left\{x_{l}\right\} \in \mathcal{S}$. ( $\mathcal{S}$ is closed with respect to effective $V$-convergence.)

In fact, $\mathrm{C} 2$ and $\mathrm{C} 3$ should be stated for multiple sequences.

A sequence belonging to $\mathcal{S}$ is called $V$-computable, and $x \in X$ is called $V$ computable if $\{x, x, \cdots\}$ is $V$-computable.

We will henceforth consider the system of the pair $\langle\mathcal{T}, \mathcal{S}\rangle$.

Remark Applications of the effective uniform space to the computability problems of some $\mathbf{E}$-discontinuous real functions are seen in [12], [13] and [21]. In the present article, we are interested in the computability property of a function sequence whose functions have different jump points, and, for that purpose, we need to work on a sequence of uniformities.

Definition 2.5 (Effective separability: [12]) 1) Suppose a $V$-computable sequence $\left\{e_{k}\right\}$ satisfies the following. For each computable sequence $\left\{x_{m}\right\}$, there is a recursive function $\mu$ such that

$$
\forall n \forall m\left(e_{\mu(n, m)} \in V_{n}\left(x_{m}\right)\right) .
$$

Then $\left\{e_{k}\right\}$ is called an effective $V$-approximating sequence of the computability structure $\mathcal{S}$.

2) Let $\left\{e_{k}\right\}$ be an effective $V$-approximating sequence. Suppose furthermore that it is dense in $X$, that is, $\forall n \forall x \exists k\left(e_{k} \in V_{n}(x)\right)$. Then the space $\langle\mathcal{T}, \mathcal{S}\rangle$ is said to be effectively $V$-separable, and $\left\{e_{k}\right\}$ is called an effective $V$-separating sequence.

We will now consider $\mathcal{U}=\left\langle\mathcal{T}, \mathcal{S},\left\{e_{k}\right\}\right\rangle$.

Note No effectivity is assumed for the association of $k$ to $n$ and $x$ in 2) above. 


\section{Model examples}

We will first quote five sequences of E-discontinuous functions from [20] (with possibly some minor modifications), since they are typical examples which explain the points of problem of our concern.

$\nu$ will subsequently represent a positive integer.

Model 1 (Gaußian function) Let $g$ be the Gaußian function, that is, $g(x)=$ the largest integer $i$ such that $i \leq x$. For simplicity, we confine ourselves to the domain $I^{+}=[1, \infty)$. Let us also consider $\left\{g_{\nu}(x)\right\}$ defined by

$$
g_{\nu}(x)=g(x) \text { if } x<\nu ;=\nu \text { if } x \geq \nu .
$$

The function $g$ can be regarded as the "limit" of the function sequence $\left\{g_{\nu}\right\}$.

In order to regard $g_{\nu}$ as continuous, it is natural to bestow the uniform topology to $I^{+}$by mutually isolating the intervals $I_{i}^{\nu}=[i, i+1), i<\nu$, and $I_{\nu}^{\nu}=[\nu, \infty)$, and conserving the interval topology $\left\{I_{i}^{\nu} \cap\left(x-\frac{1}{2^{n}}, x+\frac{1}{2^{n}}\right)\right\}$ within each of these intervals. Such a topology turns out to be a uniform topology for each $\nu$. In each interval $I_{i}$ as above, $g_{\nu}$ is E-continuous, while $g_{\mu}$ is not if $\mu>\nu$. It should be unnatural to consider $g_{\nu}$ with respect to the intervals $\left\{I_{i}^{\mu}\right\}$ when $\mu>\nu$ so that $g_{\nu}$ happens to be $\mathbf{E}$-continuous at such $\mu$. The topology determined by $I_{i}^{\nu}$ is natural for $g_{\nu}$ in the sense that it is the weakest topology with respect to which $g_{\nu}$ becomes continuous.

On the other hand, in order to regard the Gaußian function as the limit of the sequence $\left\{g_{\nu}\right\}$, it is necessary to consider all the functions $g_{\nu}$ as well as $g$ in one topology. It will be thus sensible to consider a kind of the limit of the uniformities $\left\{U_{n}^{\nu}\right\}$, in which all these functions become continuous. The limit uniformity $\left\{U_{n}^{\omega}\right\}$ turns out to be the one in which all the half-open intervals $[i, i+1), i=0,1,2, \cdots$ are mutually isolated, while each $[i, i+1)$ conserves the interval topology. $\left\{g_{2}\right\}$ is a sequence of continuous functions in the limit uniformity and the Gaußian function, the limit of this sequence, is also continuous. (This limit space is somewhat different from the "amalgamated space" for the Gaußian function in Section 6 of [12].)

Model 2 (Pulse functions) Consider a sequence of pulse functions $\left\{\psi_{\nu}\right\}$ on $I^{+}$:

$$
\psi_{\nu}(x)=\frac{1}{2^{\nu}} \text { if } \quad x=\nu ;=0 \text { if } x \neq \nu .
$$

$\psi_{\nu}$ produces a pulse of size $\frac{1}{2^{\nu}}$ at $x=\nu$. The limit of this function sequence is the constant 0 function, say $\phi . \quad \psi_{\nu}$ becomes continuous in the space which is obtained 
from the interval topology on $I^{+}$by isolating the sole point $\nu$. In order to consider the convergence of $\left\{\psi_{\nu}\right\}$ to $\phi$, we should isolate all the integers in $I^{+}$, while preserving the interval topology in the open intervals between adjacent integers. (This is the amalgamated uniformity in Section 6 of [12].)

Model 3 (Rademacher functions) Here we consider the bounded half open interval $I=[0,1)$, and the Rademacher function system $\left\{\phi_{\nu}(x)\right\}$ on $I$ (cf. [24]). The $\nu$ th function $\phi_{\nu}$ in the Rademacher system, for example, is continuous in the topology where the intervals $\left[\frac{k}{2^{\nu}}, \frac{k+1}{2^{\nu}}\right), k \leq 2^{\nu}-1$, are mutually isolated. In order to develop the Walsh analysis, we need to consider these functions in one topology. The union of the topologies above will serve the purpose.

We have elaborated on Model 3 in Sections 4 and 5 of [20] and hence we refer the reader to $[20]$.

Model 4 (Variation of tangent function) Consider the function sequence $\tau_{\nu}(x)$ defined on $\mathbf{R}$ as follows. $\tau_{\nu}(x)=0$ if $x=\frac{2 i+1}{2^{\nu}} \pi$ for some integer $i ; \tau_{\nu}(x)=$ $\tan \left(2^{\nu-1} x\right)$ otherwise. For $\nu$, if we isolate the points $\frac{2 i+1}{2^{\nu}} \pi$, then $\tau_{\nu}$ becomes continuous. (We do not consider the limit of the function sequence for this model.)

Model 5 (Cantor set) Consider the interval $I_{0}=[0,1]$. Define first a " $\nu$-decomposition" of $I_{0}$, denoted by $K_{\left\langle i_{i}, i_{2}, \cdots, i_{i}\right\rangle}^{\nu}$, where $i_{j}=0,1,2$, as follows.

$$
K_{\left\langle i_{i}, i_{2}, \cdots, i_{\nu}\right\rangle}^{\nu}=\left[\sum_{k=1}^{\nu} \frac{i_{k}}{3^{k}}, \sum_{k=1}^{\nu} \frac{i_{k}}{3^{k}}+\frac{1}{3^{\nu}}\right]
$$

if $i_{1}, \cdots, i_{\nu-1}, i_{\nu} \neq 1$;

$$
K_{\left.i_{i}, i_{2}, \cdots, i,\right\rangle}^{\nu}=\left(\sum_{k=1}^{\prime} \frac{i_{k}}{3^{k}}, \sum_{k=1}^{\nu} \frac{i_{k}}{3^{k}}+\frac{1}{3^{\nu}}\right)
$$

if $i_{1}, \cdots, i_{\nu-1} \neq 1$ and $i_{\nu}=1$;

$$
K_{\left\langle i, i_{2}, \cdots, i_{\nu}\right\rangle}^{\nu}=K_{\left\langle i_{1}, i_{2} \cdots, i_{\mu}\right\rangle}^{\mu}
$$

if there exists a $\mu \leq \nu-1$ such that $i_{1}, \cdots, i_{\mu-1} \neq 1$ and $i_{\mu}=1$.

It is obvious that, for each $\nu$, the collection of $K_{\left\langle u_{1}, i_{2}, \ldots, i_{i}\right\rangle}^{\nu}$ 's forms a decomposition of $I_{0}$, and hence, for every $x \in I_{0}$, there is a unique tuple $\left\langle i_{1}, i_{2}, \cdots, i_{\nu}\right\rangle$ such that $x \in$ $K_{\left\langle\hat{\psi}_{1}, i_{2}, \cdots, i_{i}\right\rangle}^{\nu}$. For each $\nu$, mutually isolate all the $K_{\left\langle\hat{h}_{i}, \cdots, i_{i}\right\rangle}^{\nu}$ to obtain a sequence of topologies with respect to which the characteristic functions of these intervals are continuous.

The sequence of these intervals is a process of getting the Cantor set. We will 
later deal with the characteristic functions of these decompositions.

Remark 1) Our major interest is the computability of the functions as above. Namely, we would like to consider the functions in concern as computable in the sense of computability of continuous functions in some uniform topologies (cf. Section 4 of [12]). In order to materialize this idea, we will develop the theory of effective sequence of uniformities and its effective limit according to [20]. (In [20], our emphasis was mainly on treatment of some special cases.)

2) In order to deal with the limit of a function sequence, we need to consider the functions in the sequence as well as the limit function in one topology. However, we consider it natural to assign to each function the weakest topology with respect to which the function becomes continuous. This is the reason why we work on a sequence of uniformities and its limit, and not on the limit space from the outset.

\section{Effective sequence of uniformities and its limit}

In this section, we develop a general theory of the effective sequence of uniformities and its limit.

Definition 4.1 (Effective sequence of uniformities) 1) Let $\left\{V_{n}^{\nu}\right\}=\left\{\left\{V_{n}^{\nu}\right\}_{n}\right\}_{\nu}$ be a sequence of uniformities on a set $X$ satisfying the following conditions (cf. Definition 2.2).

For each $\nu, A_{1} \& A_{2}$ holds, that is, $\cap_{n} V_{n}^{\nu}(x)=\{x\}$.

The recursive functions $\alpha_{1}, \alpha_{2}$ and $\alpha_{3}$ in $A_{3}, A_{4}$ and $A_{5}$ in Definition 2.2 depend here also on $\nu$. Thus, for example, effective $A_{4}$ stands as follows.

$$
\forall \nu, n \forall x, y \in X . x \in V_{\alpha_{2}(\nu, n)}^{\nu}(y) \Rightarrow y \in V_{n}^{\nu}(x) .
$$

Then $\left\{V_{n}^{\nu}\right\}$ will be called an effective sequence of uniformities (on $X$ ). (It is obvious that $\left\{V_{n}^{\nu}\right\}_{n}$ is an effective uniformity for each $\nu$.)

2) $\left\{x_{\nu l k}\right\}$ is said to sequentially effectively $V$-converge to $\left\{x_{\nu l}\right\}$ if there is a recursive function $\gamma$ satisfying the following.

$$
\forall \nu, l, n \forall k \geq \gamma(\nu, l, n) \cdot x_{\nu l k} \in V_{n}^{\nu}\left(x_{\nu l}\right) .
$$

We will write this property as $x_{\nu l} \simeq_{V}\left\langle x_{\nu l k}, \gamma\right\rangle$ (cf. Definition 2.3).

3) For each $\nu, \mathcal{S}_{\nu}$ will denote the computability structure on $\left\langle X,\left\{V_{n}^{\nu}\right\}_{n}\right\rangle$ (cf. Definition 2.4). The sequence $\left\{\mathcal{S}_{\nu}\right\}$ will be called an effective sequence of computability structures if the recursive functions in the definition of the computability structure (cf. Definition 2.4) depend also on $\nu$. For example, C3 stands as follows. Suppose $\left\{x_{\nu l k}\right\}_{k} \in \mathcal{S}_{\nu}$ for each $\nu$ and $l$, and $\left\{x_{\nu l}\right\} \subset X$. Suppose $\left\{x_{\nu l k}\right\}_{k}$ sequentially 
effectively $V$-converges to $\left\{x_{\nu l}\right\}$, that is, $x_{\nu l} \simeq_{V}\left\langle x_{\nu l k}, \gamma\right\rangle$ (cf. 2) above). Then $\left\{x_{\nu l}\right\}_{l} \in$ $\mathcal{S}_{\nu}$. (Any sequence belonging to $\mathcal{S}_{\nu}$ will be called $\mathcal{S}_{\nu}$-computable, or $\nu$-computable.)

4) $\mathrm{G}_{\nu}$ will denote the set of $\nu$-computable elements of $X$. If $\mathrm{G}_{\nu}$ is identical (say $\mathbf{G}_{0}$ ) for all $\nu$, then $\left\langle X,\left\{V_{n}^{\nu}\right\}_{m},\left\{\mathcal{S}_{\nu}\right\}_{\nu}\right\rangle$ is said to have a stable computability structure.

5) $\left\langle X,\left\{V_{n}^{\nu}\right\},\left\{\mathcal{S}_{\nu}\right\}\right\rangle$ is said to have a common effective $V$-separating sequence $\left\{\boldsymbol{e}_{q}\right\}$ if $\left\{e_{q}\right\} \in \mathcal{S}_{\nu}$ holds for all $\nu$, and $\left\{e_{q}\right\}$ is an effective separating sequence with respect to $\mathcal{S}_{\nu}$ for each $\nu$, where the function $\mu$ in Definition 2.5 depends also on $\nu$, that is, given a sequence $\left\{x_{\nu m}\right\}$ so that $\left\{x_{\nu m}\right\}_{m} \in \mathcal{S}_{\nu}, e_{\mu(\nu, n, m)} \in V_{n}^{\nu}\left(x_{\nu m}\right)$ holds.

We will write a system with all those structures as

$$
\mathcal{E}=\left\langle X,\left\{V_{n}^{\nu}\right\}, \alpha_{1}, \alpha_{2}, \alpha_{3},\left\{S_{\nu}\right\},\left\{e_{q}\right\}\right\rangle,
$$

and call it the system of a $\{\nu\}$-consistent sequence of uniformities.

Assumption: We will henceforth work on $\mathcal{E}$.

Corollary 1 1) A system $\mathcal{E}$ has the "computability structure intersection property," that is, $\cap \mathcal{S}_{\nu} \neq \emptyset$, since $\left\{\boldsymbol{e}_{q}\right\} \in \cap \mathcal{S}_{\nu}$.

2) $\mathcal{E}$ has the "computable elements intersection property," that is, $\cap \mathbf{G}_{\nu} \neq \emptyset$, since each $e_{q} \in \cap \mathrm{C}_{\nu}$.

Definition 4.2 (Effective limit) Given a system $\mathcal{E}$ as above, a uniformity $\left\{W_{l}\right\}$ on $X$ will be called an effective limit of $\left\{V_{n}^{\nu}\right\}$ if the following properties are satisfied.

(i) $\left\{W_{l}\right\}$ is an effective uniformity on $X$ (cf. Definition 2.2).

(ii) There are recursive functions $\varepsilon_{1}, \varepsilon_{2}$ and $\lambda$ such that

$$
\begin{gathered}
\forall l \forall x . W_{l}(x) \subset V_{\varepsilon_{2}(l)}^{\varepsilon_{1}(l)}(x) ; \\
\forall \nu \forall n \forall x . W_{\lambda(\nu, n)}(x) \subset V_{n}^{\nu}(x) .
\end{gathered}
$$

(iii) $\left\langle X,\left\{W_{l}\right\}\right\rangle$ has a computability structure $\mathcal{S}_{\omega}$ satisfying $\mathcal{S}_{\omega}=\bigcap_{\nu} \mathcal{S}_{\nu}$. (A sequence of $\mathcal{S}_{\omega}$ is called $\mathcal{S}_{\omega^{-}}$(or $\omega^{-}$)computable.)

(iv) Let $\mathbf{C}_{\omega}$ denote the set of $\omega$-computable elements of $X$. Then, $\mathbf{C}_{\omega}=\bigcap_{\nu}$ $\mathbf{C}_{\nu}$. (If $\left\{V_{n}^{\nu}\right\}$ has the stable computability structure, then $\mathbf{G}_{\omega}=\mathbf{C}_{0}$.)

(v) $\left\{e_{q}\right\}$ in $\mathcal{E}$ is an $\omega$-effective separating sequence (cf. Definition 2.5).

Note In (iii) and (iv) above, $\mathcal{S}_{\omega}$ and $\mathrm{G}_{\omega}$ are nonempty due to Corollary 1.

Definition 4.3 (Intersection property) We say that a sequence of uniformities $\left\{V_{n}^{\nu}\right\}$ has the sequential intersection property if, for $A_{3}$ in Definition 2.2, further holds that there are recursive functions $\beta$ and $\gamma$ satisfying the following: 


$$
\forall \nu_{1}, \nu_{2} \forall n, m \forall x \in X . V_{\beta\left(\nu_{1}, \nu_{2}, n, m\right)}^{\gamma\left(\nu_{1}, \nu_{2}, n\right)}(x) \subseteq V_{n}^{\nu_{1}}(\mathbf{x}) \cap V_{m}^{\nu_{2}}(x)
$$

Definition 4.4 (The effective union of $\left\{V_{n}^{\nu}\right\}$ ) Given a system $\mathcal{E}$, define $\left\{V_{l}^{\omega}\right\}$ by $V_{l}^{\omega}=$ $V_{\pi_{2}(l)}^{\pi_{1}(l)}$, where $\pi_{1}$ and $\pi_{2}$ are recursive inverse functions of a recursive pairing function $\langle p, q\rangle$ so that $l=\left\langle\pi_{1}(l), \pi_{2}(l)\right\rangle$. If $l=\langle\nu, n\rangle$, then we write $V_{l}^{\omega}$ as $V_{\langle, n\rangle}^{\omega}\left(=V_{n}^{\nu}\right)$. $\left\{V_{l}^{\omega}\right\}$ will be called the union of $\left\{V_{n}^{\nu}\right\}$.

Theorem 1 ( $\omega$-uniformity : a sufficiency condition) Given a system $\mathcal{E}$, and suppose $\left\{V_{n}^{\nu}\right\}$ has the sequential intersection property (cf. Definition 4.3). Then the union of $\left\{V_{n}^{\nu}\right\}$, viz. $\left\{V_{l}^{\omega}\right\}=\left\{V_{\langle, n, n}^{\omega}\right\}$, is the effective limit of $\left\{V_{n}^{\nu}\right\}$, and hence in particular is an effective uniformity on $X$ (cf. Definitions 2.2 and 4.2).

Proof We show (i)-(v) in Definition 4.2.

(i) $A_{1} \& A_{2}$ is obvious. $A_{4}$ and $A_{5}$ hold due to the conditions in 1) of Definition 4.1. For example, $A_{4}$ can be shown to hold as below.

$$
\forall \nu, n \forall x, y \in X . x \in V_{\left\langle\nu, \alpha_{2}(\nu, n)\right\rangle}^{\omega}(y) \Rightarrow y \in V_{\langle\nu, n\rangle}^{\omega}(x) .
$$

Notice that $V_{\left\langle\nu, \alpha_{2}(\nu, n)\right\rangle}^{\omega}(y)=V_{\alpha_{2}(\nu, n)}^{\nu}(y)$ and $V_{\langle\nu, n\rangle}^{\omega}(x)=V_{n}^{\nu}(x)$.

$A_{3}$ holds due to the sequential intersection property:

$$
V_{\left\langle\gamma\left(\nu_{1}, \nu_{2}, n, m\right), \beta\left(\nu_{1}, \nu_{2}, n, m\right)\right\rangle}^{\omega}(x) \subseteq V_{\left\langle\nu_{1}, n\right\rangle}^{\omega}(x) \cap V_{\left\langle\nu_{2}, m\right\rangle}^{\omega}(x) .
$$

(ii) Since $V_{l}^{\omega}(x)=V_{\pi_{2}(l)}^{\pi_{1}(l)}(x)$ by definition, we can take $\varepsilon_{1}=\pi_{1}$ and $\varepsilon_{2}=\pi_{2}$. Also, it holds $V_{\langle, n}^{\omega}(x)=V_{n}^{\nu}(x)$, hence we can take $\lambda(\nu, n)=\langle\nu, n\rangle$.

(iii) Define $\mathcal{S}_{\omega}=\cap_{\nu} \mathcal{S}_{\nu}$. Then $\mathcal{S}_{\omega} \neq \emptyset$ by 1) of Corollary 1. If $\left\{x_{j}\right\} \in \mathcal{S}_{\omega}$, then $\left\{x_{j}\right\} \in \mathcal{S}_{\nu}$ for all $\nu$. So, any recursive re-enumeration of $\left\{x_{j}\right\}$ is a member of $\mathcal{S}_{\nu}$ for all $\nu$, hence of $\mathcal{S}_{\omega}$.

Suppose $\left\{x_{j i}\right\} \in \mathcal{S}_{\omega},\left\{x_{j}\right\} \subset X$ and there is a recursive function $\delta$ such that

$$
\forall j \forall l \forall i \geq \delta(j, l) . x_{j i} \in V_{l}^{\omega}\left(x_{j}\right)=V_{\pi_{2}(l)}^{\pi_{1}(l)}\left(x_{j}\right),
$$

or

$$
\forall j \forall \nu, n \forall i \geq \delta(j,\langle\nu, n\rangle) \cdot x_{j i} \in V_{\langle\nu, n\rangle}^{\omega}\left(x_{j}\right)=V_{n}^{\nu}\left(x_{j}\right) .
$$

Put $\delta^{\prime}(j, \nu, n)=\delta(j,\langle\nu, n\rangle)$. Then, for each $\nu$,

$$
\forall j, n \forall i \geq \delta^{\prime}(j, \nu, n) . x_{j i} \in V_{n}^{\prime}\left(x_{j}\right),
$$

and hence $\left\{x_{j}\right\} \in \mathcal{S}_{\nu}$. This implies $\left\{x_{j}\right\} \in \mathcal{S}_{\omega}$. So, $\mathcal{S}_{\omega}$ is a computability structure.

(iv) Put $\mathbf{C}_{\omega}=\bigcap_{\nu} \mathbf{C}_{\nu}$.

(v) Recall that $\left\{\boldsymbol{e}_{q}\right\} \in \cap \mathcal{S}_{\nu}\left(=\mathcal{S}_{\omega}\right)$. If $\left\{x_{m}\right\} \in \mathcal{S}_{\omega}$, then $\left\{x_{m}\right\} \in \mathcal{S}_{\nu}$ for all $\nu$, and hence

$$
e_{\mu(\nu, n, m)} \in V_{n}^{\nu}\left(x_{m}\right)=V_{l}^{\omega}\left(x_{m}\right)
$$

where $l=\langle\nu, n\rangle$. So, $\left\{e_{q}\right\}$ is an effective $\omega$-separating sequence. 
Remark 1) In Section 6 below, we will see that the sufficiency condition in Theorem 1 is quite prevailing.

2) Theorem 1 guarantees that, under the intersection property in Definition 4. 3 , the effective limit uniformity $\left\{V_{l}^{\omega}\right\}$ exists for a system $\mathcal{E}$.

3) Some of our examples of the effective sequence of uniformities on the real line do not have the sequential intersection property. Such a sequence can nevertheless have the effective limit as we will see.

\section{A model example on the domain $[0,1)$}

In this section, we will discuss Model 3 of Section 3 at some length, as an exemplary case of an effective sequence of uniformities. This example has been discussed in detail in [20], but here it is treated as an application of Theorem 1. Other examples will be treated in Section 6.

Put $I=[0,1)$. This is the domain of our discourse in Section 5 . We assume that $\nu$ is a positive integer, and $0 \leq k \leq 2^{\nu}-1$. The sequence of uniformities in consideration will be denoted by $\left\{U_{n}^{\nu}\right\}$. In [20], we have proved some interesting interrelations between the computability problem with respect to $\left\{U_{n}^{\nu}\right\}_{n}$ for each $\nu$ and the computability problem with respect to the effective limit uniformity.

We have also worked on some function sequences defined on $I$, say $\left\{f_{\nu}\right\}$, where the index $\nu$ represents the "size of the mesh" on $I$, that is, $f$, is continuous in each mesh. Then, $\nu$ plays the role of an indicator expressing the distance of two adjacent points of discontinuity of $f_{\nu}$ (in the Euclidean topology). It then immediately follows that $\left\{U_{n}^{\nu}\right\}$ is a natural topology (the weakest topology) for $f_{\nu}$.

First we review the definition of the sequence $\left\{U_{n}^{\nu}\right\}$ and some properties from [20] for the reader's convenience.

Definition 5.1 (Neighborhoods and uniformity) Define subintervals of $I$, denoted by $I_{k}^{\nu}$, and the sequence $\left\{U_{n}^{\nu}\right\}$ as follows.

$$
I_{k}^{\nu}=\left[\frac{k}{2^{\nu}}, \frac{k+1}{2^{\nu}}\right) ; U_{n}^{\nu}(x)=I_{k}^{\nu} \cap\left(x-\frac{1}{2^{n}}, x+\frac{1}{2^{n}}\right) \quad \text { if } \quad x \in I_{k}^{\nu} .
$$

Corollary 2 (cf. [20]) 1) $\left\{U_{n}^{v}\right\}$ is a decreasing sequence with respect to $\nu$, that is, $U_{n}^{\nu+1}(x) \subseteq U_{n}^{\nu}(x)$.

2) $\left\{U_{n}^{\nu}\right\}$ is a decreasing sequence with respect to $n$, that is, $U_{n+1}^{\nu}(x) \subseteq U_{n}^{\nu}(x)$.

3) $x \in U_{n}^{\nu}(y)$ if and only if $y \in U_{n}^{\nu}(x)$ (symmetry).

4) If $x \in U_{n+1}^{\nu}(y), y \in U_{n+1}^{\nu}(z)$, then $x \in U_{n}^{\nu}(z)$ (transitivity).

Proposition 5.1 (Effective sequence : $[20]$ ) 1) $\left\{U_{n}^{\nu}\right\}$ forms an effective sequence 
of uniformities on $I$ (cf. 1) of Definition 4.1).

2) $\left\{U_{n}^{\nu}\right\}$ satisfies the sequential intersection property (cf. Definition 4.3).

1) and 2) of Proposition 5.1 have been proved in [20]. For 1), we have

$$
\alpha_{1}(\nu, n, m)=\max (n, m) ; \alpha_{2}(\nu, n)=n ; \alpha_{3}(\nu, n)=n+1 .
$$

For 2), we have

$$
\beta\left(\nu_{1}, \nu_{2}, n, m\right)=\max \left(\nu_{1}, \nu_{2}\right) ; \gamma\left(\nu_{1}, \nu_{2}, n, m\right)=\max (n, m) .
$$

Definition 5.2 ( $\nu$-computability) Let $\nu$ be an arbitrary (but fixed) positive integer, and let $\mu$ denote a finite sequence of indices, which is possibly empty. $\mu i$ denotes a finite sequence (of natural numbers) $\mu$ followed by $i$.

1) A (multiple) sequence $\left\{a_{\mu i}\right\}$ is called a $\nu$-sequence if, for each $\mu$, there exists a $k=k_{\mu} \leq 2-1$ such that $\left\{a_{\mu i}\right\}_{i} \subseteq I_{k}^{\nu}$.

2) A multiple sequence of rational numbers $\left\{r_{\mu i}\right\}$ is called a recursive $\nu$ sequence if it is recursive and is a $\nu$-sequence.

3) $\left\{a_{\mu m i}\right\}$ effectively $\nu$-converges to $\left\{x_{\mu m}\right\}$ with respect to $i$ if there is a recursive function $\alpha$ so that $i \geq \alpha(\mu, m, p)$ implies $a_{\mu m i} \in U_{p}^{\nu}\left(x_{\mu m}\right)$. We write this property as

$$
x_{\mu m} \simeq{ }_{\nu}\left\langle a_{\mu m i}, \alpha(\mu, m, p)\right\rangle
$$

or, for short, $x_{\mu m} \simeq{ }_{\nu}\left\langle a_{\mu m i}, \alpha\right\rangle$.

4) A sequence of real numbers $\left\{x_{\mu m}\right\}$ is called $\nu$-computable if there are a recursive $\nu$-sequence $\left\{r_{\mu m i}\right\}$ and a recursive function $\alpha$ as in 3 ) such that

$$
x_{\mu m} \simeq_{\nu}\left\langle r_{\mu m i}, \alpha(\mu, m, p)\right\rangle .
$$

5) A real number $x$ is called $\nu$-computable if the sequence $\{x, x, \cdots\}$ is $\nu$ computable.

Proposition 5.2 ( $\nu$-computability structure) Let $\mathcal{S}_{\text {, }}$ be the family of $\nu$-computable sequences and let $\mathcal{S}_{0}$ denote the family of $\mathbf{E}$-computable sequences. Let $\mathbf{C}_{\nu}$ be the set of $\nu$-computable elements, and let $\mathbf{C}_{0}$ denote the set of $\mathbf{E}$-computable elements.

1) $\left\{S_{\nu}\right\}$ is an effective sequence of computability structures with respect to $\left\{U_{n}^{\nu}\right\}$, and it holds that $\mathcal{S}_{\nu} \subset \mathcal{S}_{\nu+1} \subset \mathcal{S}_{0}$, where each inclusion represents a proper subset.

2) $\mathbf{C}_{\llcorner}=\mathbf{C}_{0}$.

3) A recursive enumeration of all dyadic rationals is an effective separating sequence for all $\nu$, and serves as a common effective $U$-separating sequence.

By virtue of Proposition 5.2, $\left\{U_{n}^{\nu}\right\},\left\{\mathcal{S}_{\nu}\right\}$ and $\left\{\boldsymbol{e}_{q}\right\}$ satisfy the conditions on $\mathcal{E}$, and 
hence we can now apply Theorem 1 to $\left\{U_{n}^{\nu}\right\}$ to obtain the following.

proposition 5.3 (Effective limit $\left.\left\{U_{l}^{\omega}\right\}\right) \quad\left\{U_{n}^{\nu}\right\}$ has the effective limit with respect to $\left\{\mathcal{S}_{\nu}\right\}$.

Let us denote the limit with $\left\{U_{l}^{\omega}\right\}$, or $\left\{U_{\langle\nu, n\}}^{\omega}\right\}$. By the proof of Theorem 1,

$$
U_{\langle\nu, n\rangle}^{\omega}=U_{n}^{\nu} ; \mathcal{S}_{\omega}=\bigcap \mathcal{S}_{\nu} .
$$

Remark In [20], we have presented a direct characterization of $\omega$-computable sequences, while here we have obtained the $\omega$-computability structure as an application of Theorem 1. It is a straightforward practise to show that these two notions coincide.

\section{Effectivity of models}

Returning to other models in Section 3, we assign an effective sequence of uniformities and see that it has the effective limit. At the same time, an effective separating sequence will be assigned.

Model 1 Define a uniformity $\left\{U_{n}^{1, \nu}\right\}$ on $I^{+}=[1, \infty)$ as follows.

$$
\begin{aligned}
U_{n}^{\mathbf{1}, \nu}(x) & =[i, i+1) \cap\left(x-\frac{1}{2^{n}}, x+\frac{1}{2^{n}}\right) \text { if } i \leq x<i+1 \leq \nu . \\
& =[\nu, \infty) \cap\left(x-\frac{1}{2^{n}}, x+\frac{1}{2^{n}}\right) \text { if } \quad x \geq \nu .
\end{aligned}
$$

As for the sequential intersection property (Definition 4.3), one can take the maximum of the two indices for both functions $\beta$ and $\gamma$.

A sequence of numbers from $I^{+}$, say $\left\{x_{m}\right\}$, will be called $\nu$-computable if it is an $\mathbf{E}$-computable sequence which is effectively approximated by a recursive double sequence of rational numbers, say $\left\{r_{m j}\right\}$, such that, for each $m,\left\{r_{m j}\right\}_{j} \subset\left[\iota, \iota^{\prime}\right)$ if $x \in$ $[\iota, i)$, where $\iota=i$ and $i=i+1$ for some $i<\nu$ or $\iota=\nu$ and $i=\infty$.

Let $\mathcal{S}_{1_{\nu}}$ denote the family of $\nu$-computable sequences. The set of computable elements $\mathbf{C}_{1_{\nu}}$ is exactly the $\mathbf{E}$-computable real numbers in $I^{+}$, say $\mathbf{C}_{0}$.

An effective enumeration of all rational numbers in $I^{+}$serves as a common effective separating sequence. By Theorem 1, the effective limit exists, that is,

$$
U_{l}^{1, \omega}(x)=U_{\pi_{2}(l)}^{1, \pi_{1}(l)}(x) \text {. }
$$

$\left\{U_{l}^{1, \omega}\right\}$ is effectively equivalent to the sequence $\left\{W_{j}^{1, \omega}\right\}$ below.

$$
\begin{gathered}
W_{j}^{1, \omega}(x)=[i, i+1) \cap\left(x-\frac{1}{2^{j}}, x+\frac{1}{2^{j}}\right) \text { if } x \in[i, i+1) . \\
-113-
\end{gathered}
$$


$g$ and $\left\{g_{\nu}\right\}$ are all $\omega^{\text {-continuous. }}$

Model 2 Define $\left\{U_{n}^{2, \nu}(x)\right\}$ as follows.

$$
\begin{gathered}
U_{n}^{2, \nu}(x)=\{x\}, \quad \text { if } \quad x=\nu ; \\
U_{n}^{2, \nu}(x)=\left(x-\frac{1}{2^{n}}, x+\frac{1}{2^{n}}\right) \cap[1, \nu), \quad \text { if } \quad 1 \leq x<\nu ; \\
U_{n}^{2, \nu}(x)=\left(x-\frac{1}{2^{n}}, x+\frac{1}{2^{n}}\right) \cap[\nu, \infty), \quad \text { if } \quad \nu \leq x .
\end{gathered}
$$

It is obvious that $\left\{U_{n}^{2, \nu}(x)\right\}$ forms an effective sequence of uniformities. For this model, the sequential intersection property does not hold. However, by modifying the sequence of uniformities, one can define the effective union. Namely, define another sequence of uniformities by

$$
\begin{gathered}
W_{n}^{2, \nu}(x)=\bigcap_{l \leq \nu} U_{n}^{2, l}(x), \quad \text { if } \quad x \in[0, \nu] ; \\
W_{n}^{2, \nu}(x)=U_{n}^{2, \nu}(x), \quad \text { if } \quad x \in(\nu, \infty) .
\end{gathered}
$$

$\left\{W_{n}^{2, \nu}\right\}$ is an effective sequence of uniformities. $\nu$-computable sequences are defined similarly to those in Model 1. The set of $\nu$-computable numbers is the set of $\mathbf{E}$ computable numbers. A recursive enumeration of rational numbers in $I^{+}$forms a common effective separating sequence. $\left\{W_{n}^{2, \nu}\right\}$ therefore satisfies the conditions on $\mathcal{E}$. So, Theorem 1 applies and we obtain the limit uniformity by $W_{\langle\nu, n\rangle}^{2, \omega}=W_{n}^{2, \nu}$. $\left\{\psi_{\nu}\right\}$ is $\omega$-continuous.

The space with $\left\{W_{l}^{2, \omega}\right\}$ is identical with the "amalgamated space" in Section 6 of [12]. In this space, all the nonnegative integers are isolated and the intervals between adjacent integers conserve the interval topology.

Although, for the limit alone, dealing with the sequence $\left\{W_{n}^{2, \nu}\right\}$ is sufficient, the natural topology for $\psi_{\nu}$ is $\left\{U_{n}^{2, \nu}\right\}_{n}$.

Model 4 Put

$$
U_{n}^{4, \nu}(x)=\frac{2 i+1}{2^{\nu}} \pi
$$

if $x=\frac{2 i+1}{2^{2}} \pi$;

$$
U_{n}^{4, \nu}(x)=J_{i}^{\nu} \cap\left(x-\frac{1}{2^{n}}, x+\frac{1}{2^{n}}\right)
$$

if $x \in J_{i}^{\nu}$, where $J_{i}^{\nu}=\left(\frac{2 i+1}{2^{\nu}} \pi, \frac{2 i+3}{2^{\nu}} \pi\right)$.

The sequential intersection property holds for $\left\{U_{n}^{4, \nu}\right\}$. As for an effective 
separating sequence as well as computability structure, we need a device as in [21]. The point is to assign a symbolic name to each element $\frac{2 i+1}{2^{v}} \pi$, and include it in the effective separating sequence. The $\nu$-computable sequences are defined as in the case of Model 1 except that all the points $\frac{2 i+1}{2^{\nu}} \pi$ are isolated. The conditions in Definition 4.1 are then satisfied. By Theorem 1, we can define the effective limit.

Model 5 For each $\nu$, define

$$
U_{n}^{5, \nu}(x)=K_{\langle i, i, \cdots, i \nu\rangle}^{\iota} \cap\left(x-\frac{1}{2^{n}}, x+\frac{1}{2^{n}}\right)
$$

if $x \in K_{\left\langle i, i_{1}, \ldots, i \nu\right\rangle}^{\nu}$. It is easy to show that $\left\{U_{n}^{5, \nu}\right\}$ is an effective sequence of uniformities. The notions of $\nu$-computability of sequences from $I_{0}$ (with respect to $\left\{U_{n}^{5, \nu}\right\}$ ) can be defined similarly to the case of Model 1 .

Note The definition of a computable sequence above can be extended to the definition of a multiple sequence. Precise definitions of computability structures are seen in [12], [13], [21] and [20].

\section{Computability of a function sequence in the limit}

In the effective limit, one can discuss the limit of a computable function sequence.

The definition of the "computable function" on a uniform space below is a streamlined version of the same notion in [12].

Definition 7.1 (Computable function) 1) A real-valued function $f$ from an effective uniform space with a computability structure $S$ and an effective separating sequence, say $\left\langle X,\left\{V_{n}\right\}, \mathcal{S},\left\{e_{q}\right\}\right\rangle$, is called $V$-computable if the following hold.

(i) $f$ preserves sequential $V$-computability, that is, for any $\left\{x_{i}\right\} \in \mathcal{S},\left\{f\left(x_{i}\right)\right\}$ is an $\mathbf{E}$-computable sequence of reals.

(ii) $f$ is effectively continuous in the following sense.

There is a recursive function $\gamma_{0}(q, p)$ for which

$$
y \in V_{\gamma_{0}(q, p)}\left(e_{q}\right) \Rightarrow\left|f(y)-f\left(e_{q}\right)\right|<\frac{1}{2^{p}}
$$

and

$$
\bigcup_{q=1}^{\infty} V_{\gamma_{0}(q, p)}\left(e_{q}\right)=X
$$

for each $p$. 
2) A sequence of functions, say $\left\{f_{\nu}\right\}$, is called $V$-computable if it preserves sequential $V$-computability in the sense of (i) above, viz., for any computable sequence $\left\{x_{m}\right\},\left\{f_{\nu}\left(x_{m}\right)\right\}$ is an $\mathbf{E}$-computable double sequence of real numbers, and there is a recursive function $\gamma_{0}(\nu, q, p)$ (depending also on $\nu$ ) for which (ii) above holds (for every $\nu$ ). In particular, (1) should be modified as follows.

$$
y \in V_{\gamma_{0}(\nu, q, p)}\left(e_{q}\right) \Rightarrow\left|f_{\nu}(y)-f_{\nu}\left(e_{q}\right)\right|<\frac{1}{2^{p}} \text {. }
$$

Consider next a system $\mathcal{E}$ (Section 4).

Definition $7.2\left(\{\nu\}\right.$-computable sequence of functions) Let $\left\{f_{\nu}\right\}$ be a sequence of real-valued functions on $X$.

$\left\{f_{\nu}\right\}$ is called a $\{\nu\}$-computable sequence of functions if the following three conditions hold.

(i) For each $\nu, f_{\nu}$ is $V^{\nu}$-computable, that is, $f_{\nu}$ is computable in the sense of 1) of Definition 7.1 with resepect to $\left\{V_{n}^{\nu}\right\}_{n}$.

(ii) If $\left\{x_{m}\right\} \in \cap_{\nu} \mathcal{S}_{\nu}$, then $\left\{f_{\nu}\left(x_{m}\right)\right\}$ is an $\mathbf{E}$-computable double sequence of real numbers.

(iii) There is a recursive function $\gamma_{0}$ for which hold

$$
y \in V_{\gamma_{0}(\nu, q, p)}^{\nu}\left(e_{q}\right) \Rightarrow\left|f_{\nu}(y)-f_{\nu}\left(e_{q}\right)\right|<\frac{1}{2^{p}}
$$

and

$$
\bigcup_{q=1}^{\infty} V_{\gamma_{0}(\nu, q, p)}^{\nu}\left(e_{q}\right)=X
$$

for each $\nu$.

Theorem 2 ( $V^{\omega}$-computable function sequence) Let $\left\{V_{n}^{\nu}\right\}$ be an effective sequence of uniformities in a system $\mathcal{E}$ satisfying the intersection property of Definition 4.3, and let $\left\{V_{l}^{\omega}\right\}$ be its effective limit as defined in Theorem 1. Let $\left\{f_{\nu}\right\}$ be a $\{\nu\}-$ computable sequence of functions (Definition 7.2). Then $\left\{f_{\nu}\right\}$ is computable with respect to $\left\{V_{l}^{\omega}\right\} \quad\left(V^{\omega}\right.$-computable, or $\omega$-computable).

Proof Notice first that $\left\{V_{l}^{\omega}\right\}$ is an effective uniformity as defined in Theorem 1, where $V_{\langle\nu, n\rangle}^{w}=V_{n}^{\nu}$. In order to prove the two properties in 2) of Definition 7.1, we will cite the item numbers in Definition 7.2.

$\omega$-sequential computability is guaranteed by (ii).

$\omega$-effective continuity: It suffices to define a recursive function $\eta$ such that

$$
\begin{aligned}
y \in V_{\eta(\nu, q, p)}^{\omega}\left(e_{q}\right) & \Rightarrow\left|f_{\nu}(y)-f_{\nu}\left(e_{q}\right)\right|<\frac{1}{2^{p}} \\
& -116-
\end{aligned}
$$


and

$$
\bigcup_{q=1}^{\infty} V_{\eta(\nu, q, p)}^{\omega}\left(e_{q}\right)=X
$$

With $\gamma_{0}$ in (iii), define

$$
\eta(\nu, q, p)=\left\langle\nu, \gamma_{0}(\nu, q, p)\right\rangle
$$

Then the two conditions are satisfied.

The effective convergence of a function sequence below is an improved version of the one in Definition 4.3 of [12], and is a generalization of Definition 4.1 in [8].

Definition 7.3 (Effective convergence of a function sequence) Let $\left\{f_{\nu}\right\}$ be a $V^{\omega_{-}}$ computable sequence of functions. It is said to effectively $V^{\omega}$-converge to a function $f$ if there are recursive functions $\beta(i, k)$ and $\gamma(i, k)$ satisfying the following.

(a) $x \in V_{\beta(p, q)}^{\omega}\left(e_{q}\right)$ and $\nu \geq \gamma(p, q)$ imply $\left|f_{\nu}(x)-f(x)\right|<\frac{1}{2^{p}}$.

(b) $\bigcup_{q=1}^{\infty} V_{\beta(p, q)}^{\omega}\left(e_{q}\right)=X$ for each $p$.

In fact, this definition is valid for any effective uniformity with a computability structure and an effective separating sequence.

Applications of Theorem 2 and Definition 7.3 are seen in the next section.

There is a sufficient condition for computability of the limit function in a general setting (cf. Corollary 3 in [12]).

Effective convergence of a function in the Fine metric space is discussed in [8] and in its revized version.

\section{Functions in the models}

We will speculate on the function sequences and their convergences in the models of Section 3 .

Model 1 It is obvious that $g$ is $\omega$-computable. Recall that $g_{\nu}(x)=g(x)$ if $1 \leq x<$ $\nu$ and $=\nu$ if $x \geq \nu$. From this definition, $\left\{g_{\nu}\right\}$ is $\{\nu\}$-computable, and hence by Theorem 2 it is $U^{1, \omega}$-computable. In fact $\left\{g_{\nu}\right\}$ is "uniformly" computable.

$\left\{g_{\nu}\right\}$ effectively $\omega$-converges to $g$. Since $\left\{e_{q}\right\}$ is a recursive sequence of rational numbers, one can effectively find a $\mu=\gamma_{0}(q)$ for any $q$ so that $\mu \leq e_{q}<\mu+1$. Then, in the interval $[\mu, \mu+1), g_{\mu}(x)=g(x)$ holds. Since

$$
U_{1}^{1, \omega}\left(e_{q}\right) \subset[\mu, \mu+1) \cap\left[e_{q}-\frac{1}{2}, e_{q}+\frac{1}{2}\right),
$$


putting $\beta(p, q)=\left\langle\gamma_{0}(q), 1\right\rangle$ and $\gamma(p, q)=\gamma_{0}(q)$ in Definition 7.3, we obtain (a). (b) easily follows.

Model $2\left\{\psi_{\nu}\right\}$ is $\{\nu\}$-computable since $U_{n}^{2, \nu}\left(e_{q}\right)$ is either the singleton $\left\{\boldsymbol{e}_{q}\right\}$ or an open interval on which $\psi_{\nu}$ is constant 0 . As in Model 1, there is a recursive function $\gamma_{0}$ such that $\gamma_{0}(q)=\mu$ satisfies $\mu \leq e_{q}<\mu+1$. The $\beta$ and $\gamma$ defined as follows will do for effective convergence of $\left\{\psi_{\nu}\right\}$ to the constant 0 function $\phi$.

$$
\beta(p, q)=\left\langle\gamma_{0}(q), \mathbf{1}\right\rangle ; \gamma(p, q)=\max \left(\gamma_{0}(q)+1, p+1\right) .
$$

Model 3 Yasugi and Washihara [24] have shown that the Rademacher function system is endowed with some kind of computational attributes. It has also been shown in [20] that it is a "uniform" $\omega$-computable sequence.

Here we will cite an example of Brattka which is $\omega$-computable and is effectively approximated by a "uniform" $\{\nu\}$-computable sequence of functions. (A function or function sequence is "uniformly" computable if in the definition of computability "effective continuity" can be replaced by "effectively uniform continuity.")

Example (Brattka's example: $[1]): \mu(x)$ will denote the binary representation of $x$ with infinitely many 0 's.

Let $f: I \rightarrow \mathbf{R}$ be defined as follows.

$$
f(x)=\left\{\begin{array}{c}
\sum_{i=0}^{\infty}\left(\ell_{i} \bmod 2\right) 2^{-n_{i}-\sum_{j=0}^{i=1}\left(n_{i}+\ell_{j}\right)} \\
\text { if } \left.\mu(x)=0^{n_{0}} 1^{\epsilon_{i}} 0^{n_{i}}\right]^{\ell_{1}} 0^{n_{2} \ldots} \\
\sum_{i=0}^{m}\left(\ell_{i} \bmod 2\right) 2^{-n_{i}-\sum_{j=0}^{i-1}\left(n_{j}+\ell_{j}\right)} \\
\text { if } \mu(x)=0^{n_{0}} 1^{\epsilon_{0}} 0^{n_{1}} 1^{\ell_{1}} 0^{n_{2} \ldots} 1^{\ell_{m}} 0^{\omega}
\end{array}\right.
$$

where $n_{0} \geq 0, n_{i}>0$ for $i>0$ and $l_{i}>0$ for all $i \geq 0$.

Brattka showed that the function $f$ is "Fine-computable" but is "not locally uniformly Fine-computable." $f$ is in fact not locally uniformly Fine-continuous. (See [1] for details.) However, $f$ can be effectively approximated by the function sequence $\left\{\varphi_{\nu}\right\}$ defined below (cf. [8] for details), which is "uniformly" $\{\nu\}$-computable. (Each $\varphi_{\nu}$ is "uniformly" continuous on $I_{k}^{\nu}$, and jumps at $\frac{k}{2^{\nu}}, k \leq 2^{\nu}-1$. So, $\varphi_{\nu}$ is continuous with respect to the uniformity $\left.\left\{U_{n}^{\nu}\right\}.\right) \quad\left\{\varphi_{\nu}\right\}$ is a "uniformly" $\omega^{-}$ computable sequence of functions and $f$ is an $\omega$-computable function. 


$$
\begin{aligned}
& \varphi_{1}(x)= \begin{cases}0 \quad \text { if } 0 \leq x<\frac{1}{2} \\
1 \quad \text { if } \quad \frac{1}{2} \leq x<1\end{cases} \\
& \varphi_{\nu}(x)= \begin{cases}\frac{1+(-1)^{i}}{2}+\frac{\varphi_{\nu-i}\left(2^{i}\left(x-1+\frac{1}{2^{i-1}}\right)\right)}{2^{i}} & \text { if } 1-\frac{1}{2^{i-1}} \leq x<1-\frac{1}{2^{i}} \\
\frac{1+(-1)^{\nu}}{2} & (i=1,2, \cdots, \nu-1) \\
\frac{1+(-1)^{\nu+1}}{2} & \text { if } 1-\frac{1}{2^{\nu-1}} \leq x<1-\frac{1}{2^{\nu}}\end{cases} \\
&
\end{aligned}
$$

Model 4 In each $\left(\frac{2 i-1}{2^{\nu}} \pi, \frac{2 i+1}{2^{\nu}} \pi\right), \tau_{\nu}(x)$ is effectively continuous and sequentially computable, and at $x=\frac{2 i+1}{2^{\nu}} \pi, y \in V_{n}^{\nu}(x)$ if and only if $y=x$. So, $\left\{\tau_{\nu}\right\}$ is $\{\nu\}$-computable.

Model 5 Define a function sequence $\left\{\gamma_{\nu}\right\}$ as follows. Suppose $x \in K_{\left\langle\dot{\eta}_{1}, \cdots, i_{\nu}\right\rangle}^{\nu}$.

$$
\begin{gathered}
\gamma_{\nu}(x)=0 \quad \text { if } \quad i_{1}, \cdots, i_{\nu-1} \neq 1, i_{\nu}=1 ; \\
\gamma_{\nu}(x)=1 \quad \text { if } i_{1}, \cdots, i_{\nu} \neq 1 ; \\
\gamma_{\nu}(x)=\gamma_{\mu}(x)(=0) \text { if } \exists \mu \leq \nu-1 . i_{1}, \cdots, i_{\mu-1} \neq 1, i_{\mu}=1 .
\end{gathered}
$$

It can be easily shown that $\left\{\gamma_{\nu}\right\}$ is $\{\nu\}$-computable, and hence is $\omega$-computable. In fact it is "uniformly" $\omega$-computable.

The pointwise limit, that is, $\lim _{\nu} \gamma_{\nu}(x)$ for each $x$, exists. Let us denote this limit by $c(x)$. It is obvious that $c(x)$ is the characteristic function of the Cantor ternary set. Since $\left\{\gamma_{\nu}(x)\right\}$ pointwise approximates $c(x)$, we can identify noneffectively the Cantor ternary set with the function sequence $\left\{\gamma_{\nu}\right\}$. Since the limit function $c(x)$ is not continuous with respect to the limit, the convergence can be only pointwise.

Remark In [20], we have shown that the uniformity $\left\{U_{n}^{\nu}\right\}$ of Model 3 and its diagonal $\left\{U_{n}^{n}\right\}$ are effectively topologically equivalent, and that the latter is (hence both are) effectively topologically equivalent with the Fine-metric topology. We have also shown in [22] that the metric induced from an effective uniformity by a general construction preserves effective convergence, while left it open if it preserves sequential computability. The metric obtained from the uniformity $\left\{U_{n}^{n}\right\}$ by the general construction method indeed preserves sequential computability. In [5], [6] and [8], Fine-computability and Fine-convergence are extensively studied. 


\section{References}

[ 1] Brattka, V., Some Notes on Fine Computability, JUCS, 8(2002), 382-395.

[2] Gold, E.M., Limiting recursion, JSL, 30-1 (1965), 28-48.

[3] Hayashi, S., Nakata, M., Towards Limit Computable Mathematics, International Workshop, Type 2000, Selected Papers, LNCS 2277, Springer (2002) 125-144.

[4] Kawada, Y., Mimura, Y., Outline of Modern Mathematics (in Japanese), Iwanami Shoten (1965).

[5] Mori, T., Computabilities of Fine continuous functions, Computability and Complexity in Analysis, LNCS 2064, Springer (2001) 200-221.

[6] Mori, T., On the computability of Walsh functions, TCS 284 (2002) 419-436.

[ 7 ] Mori, T., Tsujii, Y., Yasugi, M., Computability structure on metric spaces, Combinatorics, Complexity and Logic (Proceedings of DMTCS'96), Springer (1996) 351-362.

[8] Mori, T., Tsujii, Y., Yasugi, M., Fine computable functions and effective Fine convergence, CCA2005, Informatik Berichte 326-7/2005, FernUniversität in Hagen, 177-197; available also at http://www.cc.kyoto-su.ac.jp/ yasugi/page/recent.html

[9] Nakata, M., Hayashi, S., A limiting first order realizability interpretation, SCMJ Online, vol. 5 (2001) 421-434.

[10] Pour-El, M.B., Richards, J.I., Computability in Analysis and Physics, Springer-Verlag (1989).

[11] Schipp, F., Wade, W.R., Simon, P., Walsh Series, Adam Hilger (1990).

[12] Tsujii, Y., Yasugi, M., Mori, T., Some properties of the effectively uniform topological space, Computability and Complexity in Analysis, LNCS 2064, Springer (2001) 336356.

[13] Tsujii, Y., Yasugi, M., Mori, T., Sequential computability of a function-Diagonal space and limiting recursion--, Proceedings of CCA2004, ENTCS 120(2005), 187-199.

[14] Washihara, M., Computability and Fréchet spaces, Math. Jap., vol. 42 (1995) 1-13.

[15] Washihara, M., Computability and tempered distribution, Math. Jap. 50-1(1999), 1-7.

[16] Washihara, M., Yasugi, M., Computability and metrics in a Fréchet space, Math. Jap., vol. 43 (1996) 431-443.

[17] Yasugi, M., Limiting computability of discontinuous functions-significance and problems, J. of Japan Assoc. for Phil. of Sci., vol. 30, no. 2 (2003), 13-18 (in Japanese).

[18] Yasugi, M., Brattka, V., Washihara, M., Computability aspects of some discontinuous functions, Scientiae Mathematicae Japonicae (SCMJ) Online, vol. 5 (2001) 405-419.

[19] Yasugi, M., Mori, T., Tsujii, Y., Effective Properties of Sets and Functions in Metric Spaces with Computability Structure, Theoretical Computer Science, 219 (1999) 467486.

[20] Yasugi, M., Mori, T., Tsujii, Y., Effective sequence of uniformities and its effective limit, CCA2005, Informatik Berichte 326-7/2005, FernUniversität in Hagen, 301-318; available also at http://www.cc.kyoto-su.ac.jp/ /yasugi/page/recent.html

[21] Yasugi, M., Tsujii, Y., Computability of a function with jumps-Effective uniformity and limiting recursion-, Topology and its Applications (Elsevier Science), 146147(2005), 563-582.

[22] Yasugi, M., Tsujii, Y., Mori, T., Metrization of the uniform space and effective conver-

$$
-120-
$$


gence, Math. Log. Quart. 48 (2002) Suppl. 1, 123-130.

[23] Yasugi, M., Washihara, M., Computability structures in analysis, Sugaku Expositions (AMS) 13 (2000) no. 2, 215-235.

[24] Yasugi, Y., Washihara, M., A note on Rademacher functions and computability, Words, Languages and Combinatorics III, World Scientific (2003) 466-475. 